\title{
Playing a class of Game using CNN Focus on Runner Games
}

\author{
Jimut Bahan $\mathrm{Pal}^{1}$
}

${ }^{1}$ Department of Computer Science

Ramakrishna Mission Vivekananda Educational and Research Institute

A presentation for Communicative English course 


\section{Table of Contents}

Introduction

Selection of a wrong game!

Why pong failed?

Discovering a new Class of Runner Games

Results for the Dino game

Conclusion

Acknowledgements

References 


\title{
Table of Contents
}

\author{
Introduction
}

Selection of a wrong game!

Why pong failed?

Discovering a new Class of Runner Games

Results for the Dino game

Conclusion

Acknowledgements

References

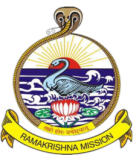




\section{Introduction}

This was a pet project during my 2nd year on St. Xavier's College!

- Motivation

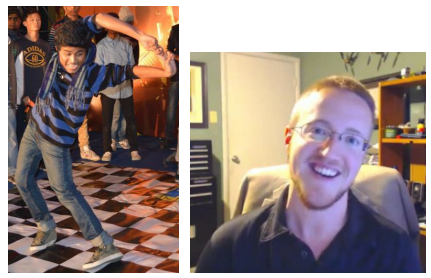

Figure: Indranil and Harrison 


\section{Introduction}

This was a pet project during my 2nd year on St. Xavier's College!

- Motivation

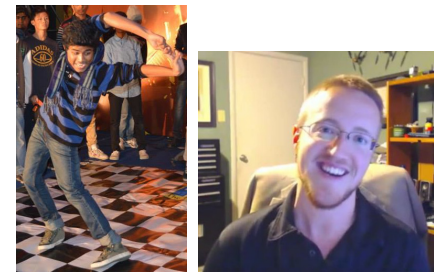

Figure: Indranil and Harrison

- CNN - to analyze visual imagery, face detection, earlier application include detection of numbers for post cards (Yann Lee Cunn) 


\section{Introduction}

This was a pet project during my 2nd year on St. Xavier's College!

- Motivation

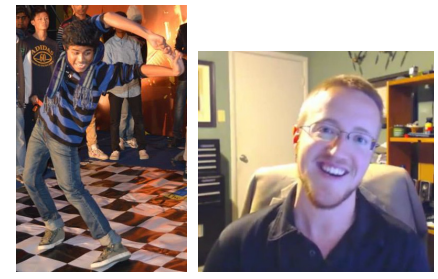

Figure: Indranil and Harrison

- CNN - to analyze visual imagery, face detection, earlier application include detection of numbers for post cards (Yann Lee Cunn)

- Cifar 10 model, $80 \%$ accuracy in 2010 


\section{Introduction}

This was a pet project during my 2nd year on St. Xavier's College!

- Motivation

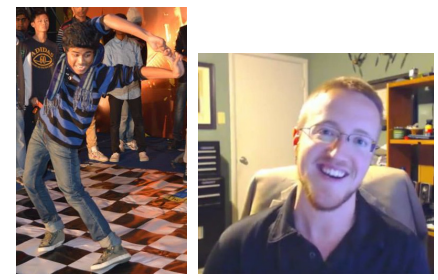

Figure: Indranil and Harrison

- CNN - to analyze visual imagery, face detection, earlier application include detection of numbers for post cards (Yann Lee Cunn)

- Cifar 10 model, $80 \%$ accuracy in 2010

- Can we make something innovative using simple technology? 


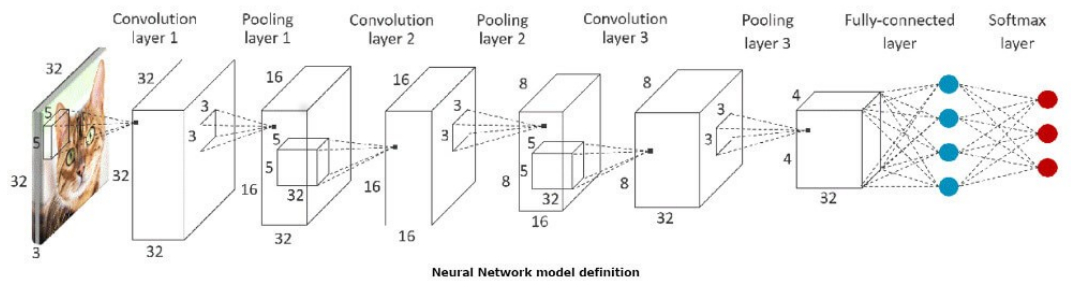

Figure: The famous CIFAR-10 model which we used for training

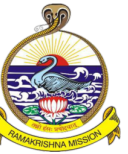




\title{
Table of Contents
}

\author{
Introduction
}

Selection of a wrong game!

Why pong failed?

Discovering a new Class of Runner Games

Results for the Dino game

Conclusion

Acknowledgements

References

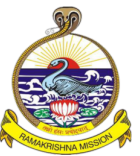




\section{Selection of a wrong game!}

The game that came to our mind - PONG!

Pong - Simplest table tennis video game from 1972 atari console.

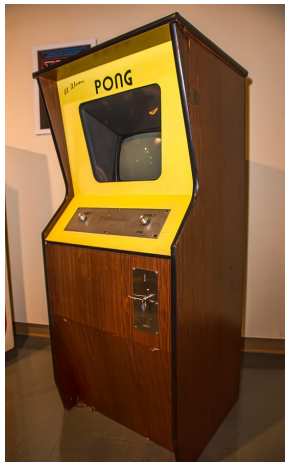

Figure: Uprighted Cabinet of Pong 


\section{Selection of a wrong game!}

The game that came to our mind - PONG!

Pong - Simplest table tennis video game from 1972 atari console.

AIl score - 1 SCORE TABLE FOR PONG-AI

Player score - o

Game ending in : 0/4000

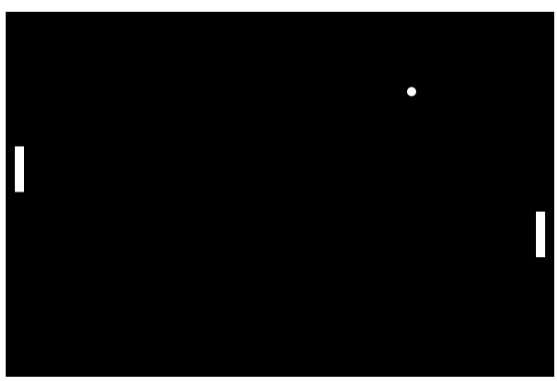

Figure: Our JS implementation of Pong 


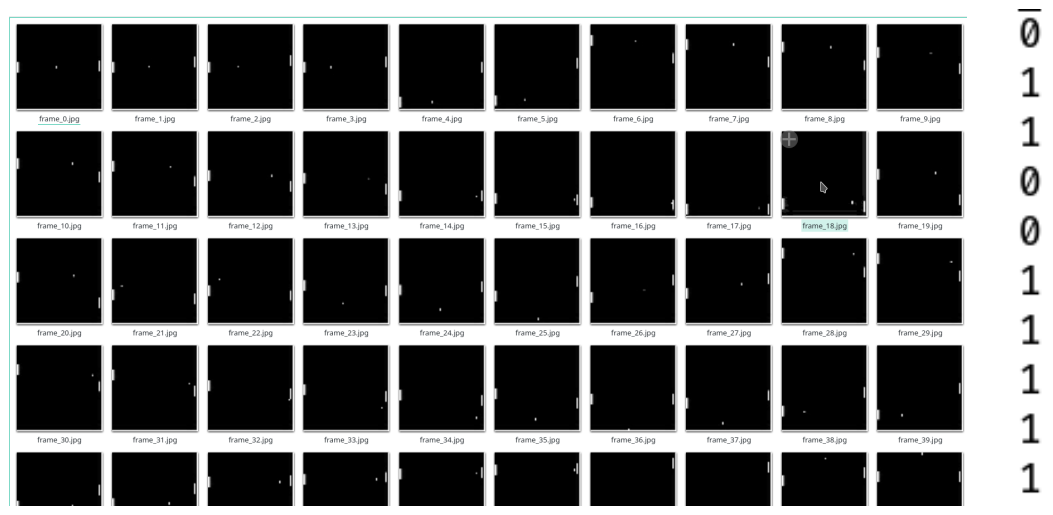

Figure: Data used in training of Pong Game: Frames and corresponding action values ( 0 - down and 1 - up) 


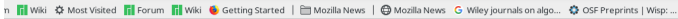

C dino_cnn.ipynb th

File Edit View Insert Runtime Tools Help

t + Text

8] $\begin{gathered}\text { tpocn } 14 / 30 \\ 1966 / 1960[-\end{gathered}$

- Epoch 15/38

1960/1960

Epoch 16/33

Epoch 17/30

196e/1960

$1968 / 1960$
Epoch 19/30

Epoch 19/38

Epoch 26/30

$1966 / 1960$ /

$1960 / 1960$

Epach 22/3日

$1960 / 1960 \mid$
Epoch 23/3日

Epoch $23 / 30$

Epoch 24/3

$1960 / 1960$
Epoch 25/30

Epoch 25/30

Epoch 26/38

196e/1960

$1960 / 1960$

Epoch $28 / 30$
$1960 / 1960$
Epoch $29 / 30$

Epoch 29/3日

Epoch $36 / 38$

- 365 19n5/step - loss: $0.1168-$ acc: 8.9582 - val_los5: 8.1777 - val_acc: 0.9096

val_loss: $\theta .1978$ - val acc: 0.9750

$[===$

- 365 1905/step - loss: $0.1139-0.00: 0.9541-$

] -

- 365 18ns/step - loss: 0.1661 - acc

1905/step - 1055: 0.0986- acc: 0.963

365 18ns/step - loss: 0.9937 - acc: 8.9653

$36519 n$ s/step - loss: 0.0938 - acc: 0.9628 -

- 365 18ns/step - los5: 0.8966 - acc: $0.9612-$

- 365 18n5/step - los5: 0.0933 - acc: 0.965

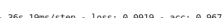

- 365 1905/step - 1055: 0. 0893 - acc 0.9684

- 365 18m5/5tep - 1055: 0.8888 - acc: 8.954 -

- 365 18m5/step - los5: 0.8888

- 375 19ns/step - loss: 0.0807 - acc: $0.9694-$

- 36s 18ns/step - loss: 0.0843 - acc: $0.9673-4$

- 36s 19ns/step - loss: 0.0787 - acc: 0.9714

- 365 19ms/step - los5: 0. 8847 - acc: $8.9699-$

val los5: 8.1772 - val acc: 0.9890

val_las5: 8.1785 - val_acc: 0.9250

val_las5: $\theta .1795$ - val_acc: 9.9000

val_lo55: $\theta .1711$ - val_acc: 9.9000

val_los5: 8.16日3 - val acc: 8.9258

val_Los5: 0.1641 - val_acc: 0.9580

val_loss: 0.1882 - val_acc: 0.9000

val_los5: $\theta .1920$ - val_acc: 0.9250

val loss: $\theta .1826$ - val acc: $\theta .925 \theta$

val_loss: 8.1934 - val_acc: 0.9250

val_las5: 8.1812 - val_acc: $0.925 \theta$

-val los5: 0.1821 - val acc: 0.9250

val loss: $\theta .1979$ - val acc: $\theta .9000$

9] Import matplotlib.pyplot as plt

print (history.history - keys())

\# sumbarize history for accuracy

plt.plot (history. history[ 'val acc'])

plt.title('nodel accuracy')

plt.ylabel ('accuracy')

plt. xlabel ('epoch')

plt. legend [ ['train', 'test'], loc='upper left']

Figure: When training in Google Collaboratory Platform 

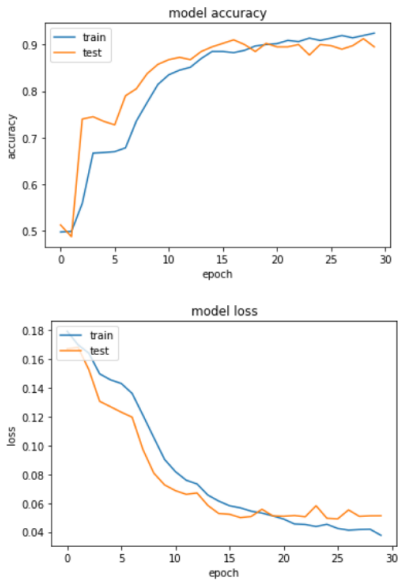

Figure: Loss and accuracy of the Pong game when trained with $2 \mathrm{~K}$ images 

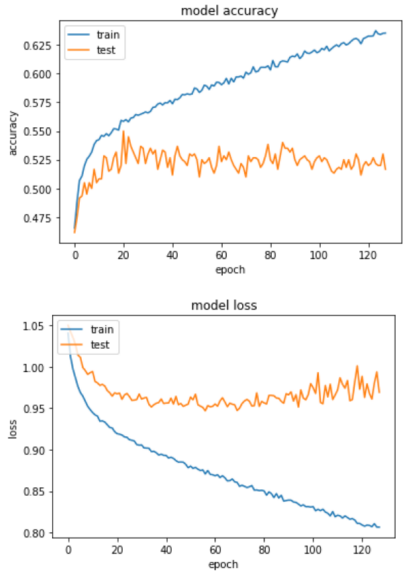

Figure: Loss and accuracy of the Pong game when trained with $30 \mathrm{~K}$ images 


\title{
Table of Contents
}

\author{
Introduction
}

Selection of a wrong game!

Why pong failed?

Discovering a new Class of Runner Games

Results for the Dino game

Conclusion

Acknowledgements

References

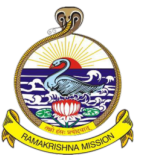




\section{Why pong failed?}

Pong's Computer counterpart is a robot not an Al, it just calculates according to coordinates of the ball. We needed human touch!

- More data more accuracy, what is wrong? 


\section{Why pong failed?}

Pong's Computer counterpart is a robot not an Al, it just calculates according to coordinates of the ball. We needed human touch!

- More data more accuracy, what is wrong?

- We trained the model using Google's free collaboratory platform, which gives free GPU and is computationally effective. 


\section{Why pong failed?}

Pong's Computer counterpart is a robot not an Al, it just calculates according to coordinates of the ball. We needed human touch!

- More data more accuracy, what is wrong?

- We trained the model using Google's free collaboratory platform, which gives free GPU and is computationally effective.

- Guess the move by looking at the Pong's picture 


\title{
Table of Contents
}

\author{
Introduction
}

Selection of a wrong game!

Why pong failed?

Discovering a new Class of Runner Games

Results for the Dino game

Conclusion

Acknowledgements

References 


\section{Runner Games}

Runner games are those kind of games which have a definite move for every instance of the environment

00045

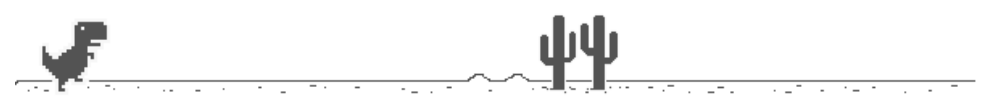

Figure: Famous Dino game! 


\section{Runner Games}

Runner games are those kind of games which have a definite move for every instance of the environment

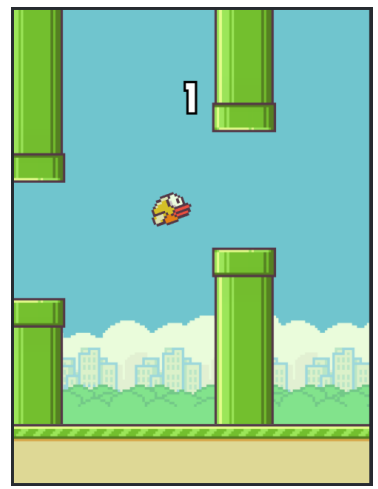

Figure: Famous flappy bird game! 


\section{Runner Games}

Runner games are those kind of games which have a definite move for every instance of the environment

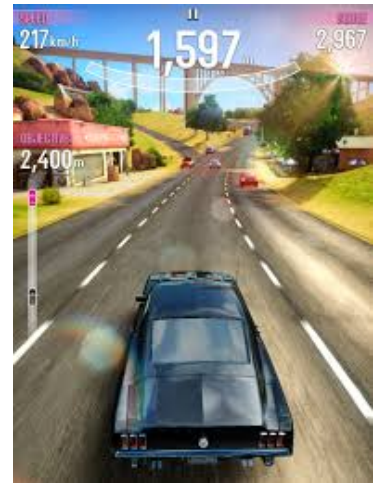

Figure: Asphalt overdrive game 


\section{Runner Games}

Runner games are those kind of games which have a definite move for every instance of the environment

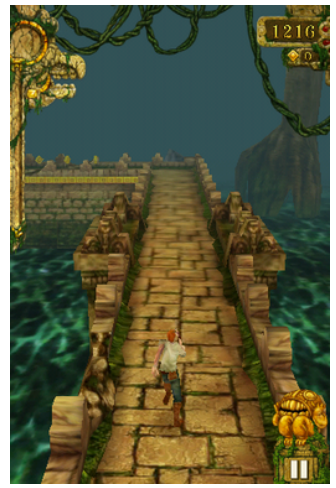

Figure: Famous Temple run game 


\section{Runner Games}

Runner games are those kind of games which have a definite move for every instance of the environment

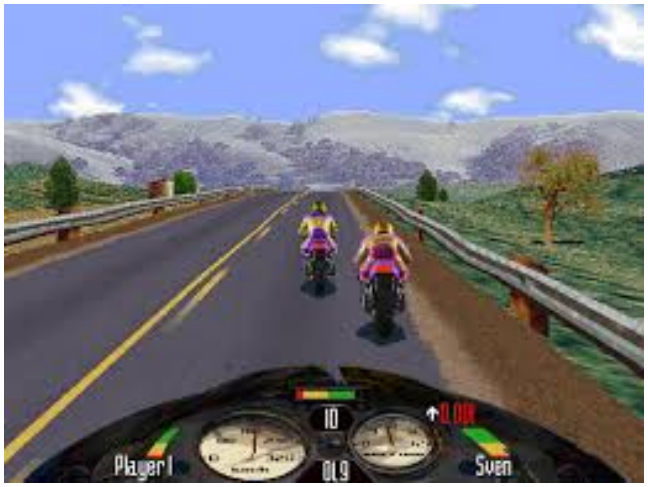

Figure: The Road Rash 


\section{Runner Games}

Runner games are those kind of games which have a definite move for every instance of the environment

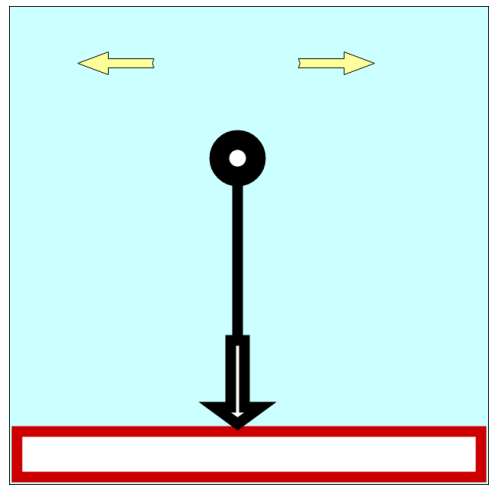

Figure: A custom made game named "Balancer" 


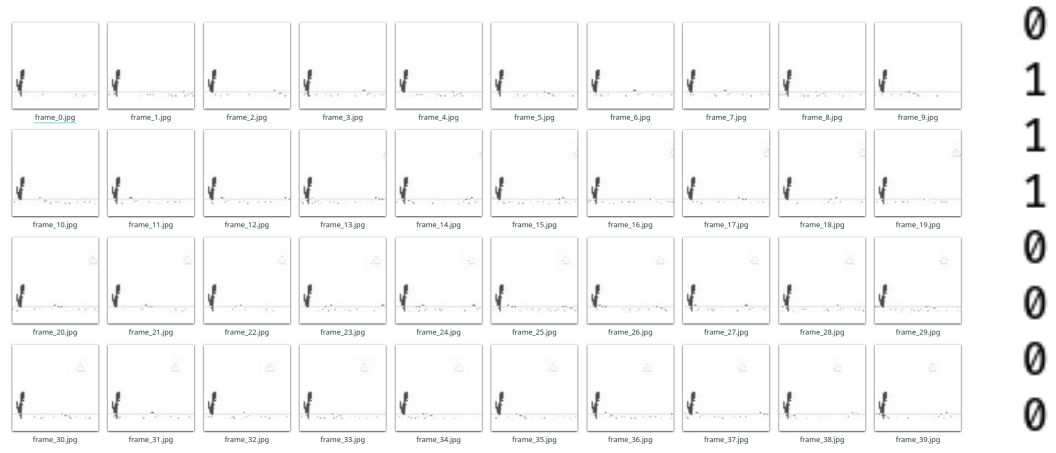

Figure: Data used in training of Dino Game: Frames and corresponding action values ( 0 - nothing and 1 - up) 


\title{
Table of Contents
}

\author{
Introduction
}

Selection of a wrong game!

Why pong failed?

Discovering a new Class of Runner Games

Results for the Dino game

Conclusion

Acknowledgements

References 


\section{Results for the Dino game}

The results obtained are satisfactory. It performed well and runs genuinely with $90 \%$ accuracy. From these results we can conclude that certain class of games performs well with just a simple technology like CNN.

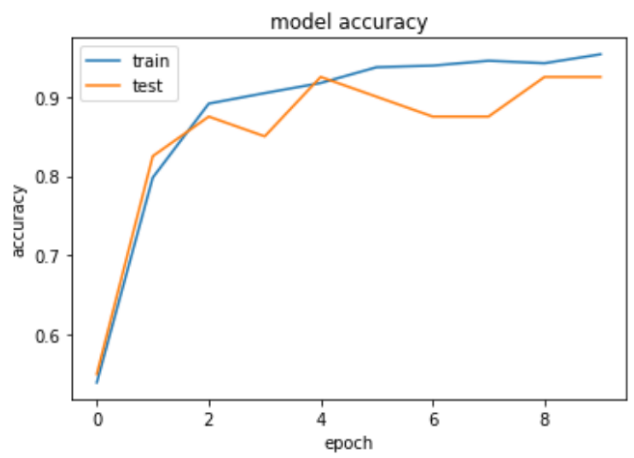

Accuracy obtained from Dino game training 


\section{Results for the Dino game}

The results obtained are satisfactory. It performed well and runs genuinely with $90 \%$ accuracy. From these results we can conclude that certain class of games performs well with just a simple technology like CNN.

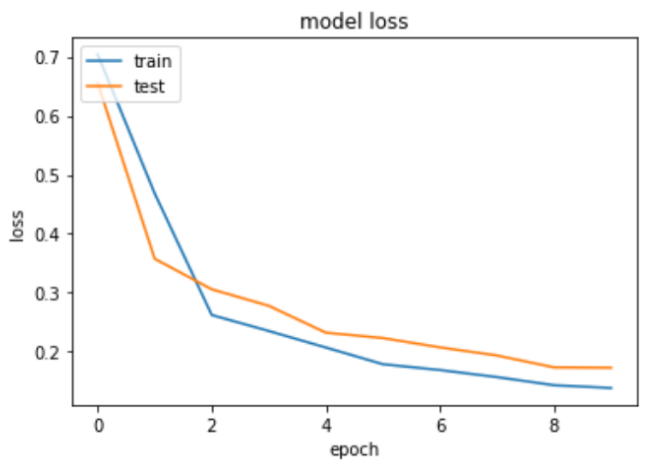

Loss from the dino game training 


\title{
Table of Contents
}

\author{
Introduction
}

Selection of a wrong game!

Why pong failed?

Discovering a new Class of Runner Games

Results for the Dino game

Conclusion

Acknowledgements

References

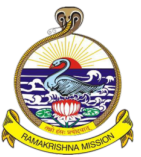




\section{Conclusion}

Modern technologies and algorithms like Reccurrent Neural Network, Reinforcement Learning, Genetic algorithms are more powerful than the method that was implemented. We will implement these in the Dino game in the near future. The YOLO works on this exact same model, i.e., extract frames from the video and predict from those taken pictures. 


\title{
Table of Contents
}

\author{
Introduction
}

Selection of a wrong game!

Why pong failed?

Discovering a new Class of Runner Games

Results for the Dino game

Conclusion

Acknowledgements

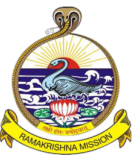




\section{Acknowledgements}

I acknowledge the help recieved from Tamal Maharaj, Prof. Janardan Ghosh, Indranil Das and Harrison Kinseley for their suggestions and discussions. 


\title{
Table of Contents
}

\author{
Introduction
}

Selection of a wrong game!

Why pong failed?

Discovering a new Class of Runner Games

Results for the Dino game

Conclusion

Acknowledgements

References

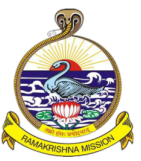




\section{References}

- Pal, J.B., (2019), Playing a class of games using CNN, Blog in github pages, https://jimut123.github.io/blogs/cnn_games_ai.html available on the web, last accessed on 14-11-2019 .

- Kinseley, H., (2017), Python Plays GTA V, Tutorial in https://pythonprogramming.net/game-frames-open-cvpython-plays-gta-v/ available on the web, last accessed on 14-11-2019 . 
Thank You 